\title{
Germanica
}

\section{Rythmes, ornements et masses : Siegfried Kracauer et l'orchestration du pouvoir}

Graeme GILLOCH : Rhythmus, Ornament und Massen: Siegfried Kracauer und die Orches-trierung der Macht

Graeme GILLOCH : Rhythms, Ornaments and Masses: Siegfried Kracauer and the Orchestra-tion of Power

\section{Graeme Gilloch}

Traducteur : Antonella Di Trani et Olivier Agard

\section{OpenEdition}

\section{Journals}

Édition électronique

URL : https://journals.openedition.org/germanica/8551

DOI : 10.4000/germanica.8551

ISSN : 2107-0784

Éditeur

Université de Lille

Édition imprimée

Date de publication : 1 juin 2020

Pagination : 85-100

ISBN : 978-2-913857-45-2

ISSN : 0984-2632

Référence électronique

Graeme Gilloch, «Rythmes, ornements et masses : Siegfried Kracauer et l'orchestration du pouvoir », Germanica [En ligne], 66 | 2ème trimestre 2020, mis en ligne le 02 janvier 2022, consulté le 08 janvier 2022. URL : http://journals.openedition.org/germanica/8551 ; DOI : https://doi.org/10.4000/ germanica.8551 


\title{
Rythmes, ornements et masses : Siegfried Kracauer et l'orchestration du pouvoir
}

\author{
Graeme Gilloch \\ Lancaster University
}

Cet article est une contribution au regain d'intérêt tant attendu pour les travaux de Siegfried Kracauer, mais aussi pour des textes relatifs à la Théorie critique largement négligés, et qui se sont surtout focalisés sur l'autoritarisme, les agitateurs fascistes aux États-Unis 1 et l'antisémitisme. Notre article vise à améliorer la compréhension et l'appréciation de Totalitäre Propaganda en situant ce texte dans une constellation d'études de la fin des années $1920^{2}$. Il s'agit d'écrits qui examinent le caractère, les prédilections, les dispositions et les goûts de ce que Kracauer avait identifié, avec perspicacité, comme une classe moyenne montante et vivant en milieu urbain, composée d'employés de bureau de tous genres : secrétaires et dactylographes, fonctionnaires subalternes, modestes bureaucrates et commerçants ${ }^{3}$. Ces nouvelles couches allaient s'avérer être les plus sensibles à la propagande totalitaire. Les discours et les mises en scène

1. - Norbert Guterman, Leo Löwenthal, Prophètes du mensonge : Étude sur l'agitation fasciste aux États-Unis, Paris, La Découverte, 2019.

2. - Siegfried Kracauer, Totalitäre Propaganda, (hg. und mit einem Nachwort versehen von Bernd Stiegler; unter Mitarbeit von Joachim Heck und Maren Neumann), Berlin, Suhrkamp, 2013.

3. - Siegfried Kracauer, Les Employés. Aperçus de l'Allemagne nouvelle [1929], (trad. de l'allemand par Claude Orsoni ; éd. et présenté par Nia Perivolaropoulou), Paris, Éditions de la MSH, 2004.

GERMANICA, 2020, LXVI, pp. 85 à 100. 
fabriqués par le national-socialisme allaient jouer sur leurs penchants, toucher leurs anxiétés, aspirations et fantasmes. Elles en sont donc venues à constituer les « masses » qui, en fin de compte, ont faussement été désignées comme Volksgemeinschaft (communauté du peuple).

Situer Totalitäre Propaganda dans le contexte de textes antérieurs de Kracauer nous amène inévitablement à mettre de côté quelques aspects importants du texte. S'il existe des omissions dans ce qui suit, l'accent que j'ai mis sur le thème fondamental des masses est néanmoins justifié par le fait que, lorsque Horkheimer et Adorno ont, sans surprise, décliné de publier le texte intégral dans Zeitschrift für Sozialforschung, soidisant en raison de sa longueur, la requête de Kracauer de publier au moins la section sur les masses et la conclusion resta lettre morte 4 . J'affirmerais que c'était précisément dans ces pages que lui-même voyait la substance et les fondements de son raisonnement d'ensemble.

\section{Les inquiétudes des cols blancs}

Publié en premier lieu sous la forme d'une série de feuilletons dans la Frankfurter Zeitung 5 en 1929, l'ouvrage de Kracauer Les Employés. Aperçus de l'Allemagne nouvelle est un récit quasi-ethnographique singulier décrivant la force de travail des cols blancs de la capitale. Les Employés est sans doute un texte relativement familier, je vais donc me limiter ici à traiter les trois questions qui me semblent être les plus pertinentes à propos de Totalitäre Propaganda. C'étaient après tout, comme le souligne Kracauer en citant Les Employés, ces membres obéissants et disciplinés des classes moyennes (Mittelschichten), mobilisés dans les circonstances de la crise économique ${ }^{6}$ qui allaient devenir la véritable étoffe du national-socialisme ${ }^{7}$.

Tout d'abord en tant qu'employés de bureau, ils sont complètement empêtrés dans la structure organisationnelle, les contraintes et les procédures de ce que l'on nomme en termes courants de la Ratio, la logique de la raison instrumentale (Zweckrationalität) qui gouverne la vie bureaucratique. En effet, les employés ne sont pas seulement inscrits dans les hiérarchies technocratiques produites par la discipline, l'expertise et la rationalisation caractéristique de l'autorité légale-rationnelle wébérienne, ils en sont aussi les dépositaires en ce sens qu'ils ont pour tâche d'administrer et de réglementer la vie des autres. Étant eux-mêmes

4. - S. Kracauer, Totalitäre Propaganda, op. cit., p. 131-156 sq.

5. - D'où son élaboration avant que Kracauer n'arrive à Berlin en 1930 et devienne l'éditeur des pages culturelles du journal.

6. - S. Kracauer, Totalitäre Propaganda, op. cit., p. 108.

7. - Kracauer affirme sans équivoque : «[...] les classes moyennes en déclin forment les bases du national-socialisme» ( (Die gesunkenen Mittelschichten bilden die natürliche Basis des Nationalsozialismus. »), ibid., p. 125. Sauf mention contraire, nous traduisons. 
entièrement subordonnés aux logiques des strictes lois impersonnelles, ils demandent en retour un asservissement inconditionnel de leurs clients, la bonne exécution et la circulation ponctuelle de formalités administratives standardisées et normalisées.

Prises dans la routine quotidienne, il n'est peut-être pas étonnant que les masses de salariés cherchent des compensations dans l'illusion offerte par les distractions et les industries culturelles de la ville. Le divertissement servi comme une évasion du réel, pouvait impliquer le type de fantasme romantique et mélodramatique (le fils du patron qui tombe amoureux de la secrétaire humble et timide) que Kracauer rejette avec mépris dans son essai intitulé (de façon condescendante) « Les petites vendeuses vont au cinéma » $(1927)^{8}$. Il n'est peut-être pas surprenant que les personnalités charismatiques et les passions interdites, que l'on trouve sur des écrans de cinéma ou dans les pages de la littérature de colportage, puissent être dotées d'une séduction et d'un attrait particulier pour ceux dont la vie active est nécessairement vouée à l'application résolument dépassionnée de règles et régulations impersonnelles prédéterminées. Le « Monde du calicot $»^{9}$ qui a été créé par l'essor de l'industrie du film allemand ou bien encore les pseudo-réalités évoquées par des Prophètes du mensonge boursouflés offre non seulement une évasion mais aussi une consolation contre l'ennui et la trivialité des vies les plus ordinaires.

De façon plus significative, ces classes moyennes (Mittelschichten) occupent un espace intersticiel (Zwischenraum), un des termes préférés de Kracauer, dans la structure des classes sociales elle-même. Cette classe moyenne est constituée de personnes qui demeurent sur un seuil face auquel elles hésitent. Les membres de cette classe ne possèdent pas, et n'aspirent pas nécessairement au capital culturel et social de la bourgeoisie cultivée (Bildungsbürgertum) ou de la haute bourgeoisie et des professions libérales, dont ils envient et critiquent à la fois les statuts, les revenus et les privilèges; on songe alors aux patriciens snobs tels que l'écrivain et critique espagnol José Ortega Y Gasset. En même temps, ils se dissocient de façon véhémente de la classe ouvrière, du monde des syndicats, de la main d'œuvre et de la solidarité prolétarienne. Le fait d'être démunis, d'un côté, de l'assurance hautaine propre à la bourgeoisie, et de l'autre, d'une conscience authentique de classe, met les cols blancs dans une position délicate et instable : ils n'ont ni le statut d'inclus (face à l'establishment et aux élites envers lesquelles ils

8. - Siegfried Kracauer, "Les petites vendeuses vont au cinéma », in : Id., L'Ornement de la masse. Essais sur la modernité weimarienne, trad. de l'allemand par Sabine Cornille, texte édité par Olivier Agard et Philippe Despoix, Paris, La Découverte, 2008, p. 255-268.

9. - S. Kracauer, « Monde du calicot», in : Id., L'Ornement de la masse, op. cit., p. $247-254$. 
nourrissent du ressentiment) ni celui d'exclus (pour lesquels ils expriment un mépris non déguisé) ${ }^{10}$.

Kracauer diagnostique ce complexe simultané d'infériorité et de supériorité comme l'indice d'une condition particulière : le fait d'être « spirituellement sans abri » ${ }^{11}$. Il s'agit là de couches socio-économiques dépourvues de toute conscience de classe, incapables de reconnaître de véritables intérêts communs, et par là-même incapables d'envisager des formes d'action collective. C'est la myopie des employés de bureau au regard de leur position structurelle qui les rend trop vulnérables à l'illusion d'une société sans classe et aux fausses promesses des personnalités autoritaires, surtout dans les moments où les crises économiques menacent la mobilité sociale et économique. Dans ces moments-là, les employés salariés doivent faire face à la perspective redoutable de la " prolétarisation », à ce qu'ils perçoivent comme l'ignominie du travail ouvrier et aux outrages de la bourse de l'emploi. La promesse d'une Volksgemeinschaft était d'offrir un refuge commun et commode pour toutes ces « âmes sans abri » au milieu d'un tel désespoir.

\section{Le rythme de la Ratio}

L'essai de Kracauer «L'ornement de la masse », la deuxième partie de notre triptyque textuel des années 1920, s'ouvre avec la revendication épistémologique et méthodologique suivante :

Le lieu qu'une époque occupe dans le processus historique se détermine de manière plus pertinente à partir de l'analyse de ses manifestations discrètes de surface, qu'à partir des jugements qu'elle porte sur elle-même. Ceux-ci, en tant qu'expression des tendances du temps, ne sont pas des témoignages concluants sur l'état d'esprit global du siècle. Les premières, par leur caractère inconscient, donnent directement accès au contenu fondamental de la réalité existante. Inversement, leur interprétation est liée à la connaissance de celui-ci. Le contenu fondamental d'une époque et ses mouvements demeurés inaperçus s'éclairent mutuellement ${ }^{12}$.

Dans cette dialectique de la superficialité et de la profondeur, Kracauer précise que l'étude de phénomènes apparemment superficiels ne doit pas se confondre avec une étude superficielle. En effet, ces manifestations en apparence triviales et insignifiantes sont les points essentiels, peut-être les seuls points d'accès, à ce qui se dissimule sous la surface, aux conditions fondamentales qui règnent dans une société à un moment donné. Le choix que fait Kracauer de se concentrer sur des « manifestations discrètes de

10. - S. Kracauer, Les Employés, op. cit., p. 137.

11. - S. Kracauer, Totalitäre Propaganda, op. cit., p. 108.

12. - S. Kracauer, «L'ornement de la masse », in : Id., L'Ornement de la masse, op. cit., p. 60-71, ici p. 60 . 
surface $»^{13}$ est spécifiquement lié au monde du divertissement populaire : Kracauer part de l'exemple d'une troupe de danse de Grande-Bretagne, les Tiller Girls, dont les performances à l'époque faisaient sensation dans les nombreux théâtres de Berlin et ailleurs. Dans leurs numéros de danse aux rythmes élaborés et aux chorégraphies parfaitement synchronisées, les Tiller Girls incarnaient le sort de la physis humaine dans la modernité capitaliste, en illustrant une forme de subordination à une nouvelle logique sociale et culturelle sous-jacente, ainsi que le triomphe d'une esthétique de la machine, caractéristique d'un monde désenchanté. Les routines de danse, le mot routine étant en soi révélateur, rassemblaient des audiences admiratives devant le spectacle de l'homogénéité et de l'hégémonie de la Ratio. Les Tiller Girls ne témoignent pas simplement de la vacuité des distractions de la culture moderne mais de façon plus significative, elles expriment et annoncent le sort de la modernité individuelle sous le capitalisme. Ces éléments sont de la plus grande pertinence pour l'étude ultérieure Totalitäre Propaganda. Tout d'abord il y a le problème fondamental de la dédifférenciation et de la désindividualisation, lié au processus même de massification. Dans les différents numéros des Tiller Girls on ne voit pas des danseuses singulières et distinctes se mouvant de façon spontanée et indépendante mais plutôt une série de « [...] groupes indissolubles de jeunes filles [...] $\gg^{14}$. Ayant été choisies précisément pour leur ressemblance (stature, forme du corps et teint) et habillées avec des costumes identiques, elles ne forment pas un groupe d'individus reconnaissables avec un talent commun mis au service de la danse mais plutôt une totalité désindividualisée, une masse. Du fait de la multiplication et la synchronisation, l'individu comme être unique est réduit à sa signification la plus mince. On ne peut pas dire que la totalité est ici supérieure à la somme des parties : la partie n'a pas de sens en elle-même.

En second lieu, il y a dans cette danse un passage de l'organique/ biologique au mécanique, à l'automatique. L'effet visuel et le principe esthétique à l'œuvre dans les performances des Tiller Girls impliquent la sujétion des corps dansants, non pas à des rythmes innés et naturels mais plutôt à des répétitions mécaniques et des tempi accélérés. Le corps de la danseuse comme élément ne se livre pas à une performance mais à un exercice fonctionnel exécuté à toute vitesse avec une régularité robotique. L'improvisation et la spontanéité sont évacuées au profit d'une coordination méticuleuse et prédéterminée. Rien n'est laissé au hasard dans cette chorégraphie. Kracauer écrit :

La structure de l'ornement de masse reflète celle de la situation d'ensemble aujourd'hui. Étant donné que le principe du procès de production

13. - Ibid.

14. - S. Kracauer, « L'ornement de la masse », op. cit., p. 60. 
capitaliste ne relève pas purement de la nature, il doit faire éclater les organismes naturels qui sont pour lui des moyens ou des obstacles. Communauté populaire et personnalité s'effacent devant l'exigence de calculabilité ; l'homme comme simple particule de la masse peut sans difficulté grimper dans les statistiques et servir les machines ${ }^{15}$.

Les Tiller Girls sont des « corps dociles » dansant au rythme de la Ratio. Cela nous amène à un troisième aspect important : «L'ornement est soi-même sa propre fin ${ }^{16}$. En tant que « reflet esthétique »de la logique capitaliste - entendue comme la partie de la raison qui se contente d'être efficace, tout en restant indifférente quant aux fins, en d'autres termes, la raison instrumentale ou Ratio -, les performances des danseuses sont subordonnées à la production de modèles et à la formation de figures qui n'ont d'autres fins qu'elles-mêmes. Leurs corps deviennent un instrument au service de ce que Kracauer désigne comme de pures abstractions. Les figures et les ornements se suffisent à euxmêmes. Les danseuses, dociles, sont purement décoratives. Kracauer reconnaît à la fois la nouveauté et le sens de cette insignifiance radicale :

Ces constellations ne visent rien en dehors d'elles-mêmes, et la masse d'où elles s'élèvent n'est pas, comme la compagnie, une unité morale. On ne peut même pas aborder ces figures comme des ornements secondaires accompagnant le travail de discipline de l'éducation physique. Les unités de girls s'exercent plutôt à produire un grand nombre de lignes parallèles, et on aimerait un entraînement de masses humaines les plus larges possible, afin d'obtenir un motif de dimensions inouïes. À la fin, il y a l'ornement, et les structures porteuses de substance se sont vidées pour constituer son espace $\operatorname{clos}^{17}$.

L'abstraction (Abstraktheit) triomphante des corps obéissants et subordonnés aux logiques de la production capitaliste est comme un spectacle qui permet les aperçus les plus parlants sur la condition de l'existence moderne en tant que biopolitique disciplinaire et martiale ${ }^{18}$. Ainsi, loin de fustiger sans merci sa vacuité, Kracauer en vient à reconnaître

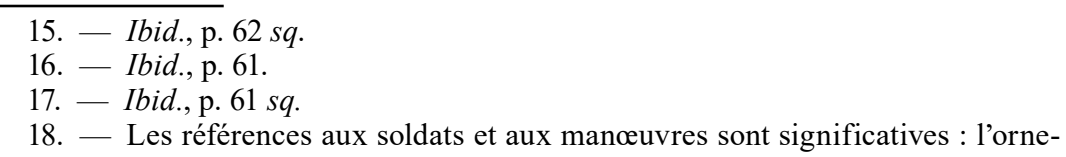
ment de masse atteint son apothéose esthétique dans les dispositifs chorégraphiques militaires qui étaient centraux dans les rassemblements fascistes et national-socialistes. Il est aisé de voir comment ces performances alignées et synchronisées et ces revues sont devenues des parades et rassemblements, comment les sequins et les plumes ont été transformés en svastikas et drapeaux. L'« ornement de masse » est donc le noyau de la compréhension de la propagande totalitaire de Kracauer et de « l'esthétisation de la politique » de Benjamin. 
l'ornement de la masse comme l'expression esthétique la plus sensible, et comme l'expression de l'esthétique même, de son temps :

Si faible que soit la valeur qu'on attribue à l'ornement de masse, il se situe, d'après son degré de réalité, au-dessus de ces productions artistiques qui continuent à cultiver dans des formes du passé de grands sentiments périmés; ne signifierait-il rien d'autre ${ }^{19}$.

Enfin, il se pose aussi le problème du positionnement et du point de vue du public. Ce qui se produit dans les coulisses n'est pas insignifiant. Il y a à cet égard des tendances contradictoires, d'un côté la sensation de l'immersion individuelle dans la multitude de spectateurs est un spectacle en soi, et au milieu de la masse on se sent « chez soi », que ce soit au théâtre ou au milieu de la foule des stades. De l'autre côté, les motifs composés par les corps dansants sont parfois uniquement reconnaissables, lisibles à partir d'une certaine distance. En effet, ils sont conçus dès le début en fonction d'un point de vue particulier et idéal, celui du spectateur parfaitement placé, qui trône en surplomb de la performance. Anticipant le regard en plongée de la caméra, la configuration des danseurs est faite pour être appréciée à partir d'un point de vue élevé et privilégié. Le spectateur de l'ornement de la masse est positionné soit comme une fourmi soit comme un dieu, comme un suiveur ou comme un meneur. Alors, comment l'étude sur Les Employés, brièvement résumée plus haut, et « L'ornement de la masse » de Kracauer sont-ils à la base de la notion tardive des « masses » dans l'étude de la propagande totalitaire? Il existe un certain nombre de thèmes clés qui font le lien.

D'abord de façon significative, ces deux textes mettent en évidence le processus de désindividualisation et de dépersonnalisation impliquant des modes de fracturation et de fragmentation, ainsi que la dissolution d'autres liens (potentiels) et d'autres formes d'association. Le corps individuel et le corps social sont brisés. L'individu est réduit à, et préservé en tant qu'atome « spirituellement sans abri », ou « particule de masse » (« Massenpartikel ${ }^{20}$ ). La masse est alors un asile, une institution totale selon la définition de Goffmann, vouée à la profonde mortification et à la reconstruction du soi. Dans une symbiose d'autoritarisme et de flagornerie, la personnalité du leader, son charisme sans cesse réitéré, son caractère unique et son autonomie sont d'autant plus valorisés, vénérés, quand on est soi-même dépourvu de personnalité. La Persönlichkeit, note Kracauer, « est un des pôles des régimes totalitaires, la masse en est l'autre » ${ }^{21}$.

19. - Ibid., p. 64.

20. - S. Kracauer, Totalitäre Propaganda, op. cit., p. 80.

21. — « [...] ist der eine Pol des totalitären Regines; die Masse der andere », ibid., p. 95 sq. 
Ces deux textes suggèrent à quel point ce déclin et même cette éventuelle destruction du sujet sont suivies par des processus de reconstruction et de reconfiguration sous la forme de nouveaux dispositifs qui représentent et créent des structures, des organisations, des formes de connexions, des modèles, des totalités. En effet, ce sont précisément ces processus en apparence homogènes de dé- et re-composition dans de nouvelles combinaisons qui sont mises en scène par les Tiller Girls, sans effort apparent. Comme si la réalité persistante de l'antagonisme des classes sous le capitalisme avait été éradiquée comme par magie, la nouvelle totalité est invoquée et construite sous la forme d'une entité illusoire : la Volksgemeinschaft. Celle-ci n'est pas seulement le principe organisateur dans lequel l'individu est subsumé, mais c'est le vrai but et la mission que l'individu se devait de réaliser. Les schémas abstraits de l'ornement sont formés par les actions concertées des masses, ces dernières n'ayant d'autres finalités qu'elles-mêmes. Il n'y a pas de solos. La masse demande de la conformité, elle ne supporte pas la différence, la dissidence politique. La masse est une machine disciplinaire régie par l'auto-discipline. La masse est narcissique, elle s'admire, cherche sa propre image, son propre visage dans les nouveaux médias de masse émergents. Elle cherche à lire des contenus sur elle-même dans les journaux et les magazines, elle cherche à se regarder dans les théâtres et sur scène afin de s'admirer elle-même sur l'écran. La masse est fascinée par ses propres traits, elle est son propre ornement, son propre spectacle.

Enfin la notion de spectacle est elle-même trop limitée et restrictive. Il est important d'aller au-delà de la notion de spectacle et de considérer la dimension d'incarnation de la masse ${ }^{22}$. C'est ici que l'importance du rythme apparaît. Pour Kracauer, le rythme est à la fois corporel - les corps bougent ensemble dans le temps et l'espace -, et entraînant. Comment peut-on ne pas danser en rythme sur la scène, applaudir à contretemps au milieu du public, exister de façon décalée par rapport à son voisin ? Le sentiment de faire partie de la masse ne s'explique pas seulement par le fait d'être regardé d'en bas ou d'en haut, ou en tant que spectacle dans les actualités cinématographiques. La masse implique une proximité physique et le sentiment de faire partie de quelque chose de plus grand, de plus étendu, de plus significatif que son propre moi. La masse se ressent elle-même comme quelque chose de plus grand que la somme de ses parties. La masse est selon la description célèbre de Raymond Williams, une culture, la masse est une « structure de sentiments $»^{23}$.

22. - Cela est certainement important pour Ortega y Gasset (1930-1994) : il voit l'arrivée des masses tout d'abord comme une occupation physique des sites et espaces qui étaient autrefois réservés aux privilégiés.

23. - Raymond Williams, Marxism and Litterature, Oxford, Oxford UP, 1977. 


\section{Le paysage urbain symphonique}

Telles les routines de danse des Tiller Girls, le film Berlin, symphonie d'une grande ville de Walter Ruttmann, est une autre « manifestation discrète de surface $»$ de son temps ${ }^{24}$. Ses images décrivent et représentent les structures disparates et diverses, des scènes, sites et vues d'un jour ordinaire de début de printemps dans la ville contemporaine de Berlin, une ville qui s'est énormément agrandie au cours des décennies précédentes en termes de population et du fait d'un accroissement continu de tous les secteurs : industries, monde des affaires, commerce, administration publique et loisirs. Commençant par l'arrivée d'un train dans la gare d'Anhalt au petit matin, le film de Ruttmann recourt à une grande variété de technologies et de techniques cinématographiques innovantes, en variant les enchaînements et la vitesse dans le montage, en utilisant de façon pionnière des caméras mobiles, en recourant à des angles de prise de vue inhabituels, des techniques de superpositions, afin de capter les rythmes variés et entrecroisés du monde métropolitain quotidien, en particulier les nouveaux rythmes du travail, de la production, du trafic, des transports en commun, et de la foule qui grouille, comme autant de phénomènes contemporains dynamiques et complexes. Pour Ruttmann, la ville était une totalité kaléidoscopique, un assemblage de différentes parties interconnectées et fonctionnelles intégrant les humains, les machines et les infrastructures dans un « [...] organisme artificiel imprégné de sa propre vie [...] $»^{25}$.

Comme le souligne Kracauer, c'était Carl Mayer, qui se trouve aussi être l'auteur du scénario du Cabinet du Dr Caligari (Robert Wiene, 1920), qui a eu l'idée originale d'une symphonie urbaine en $1925^{26}$. Frustré par les restrictions et irrité par les artifices des studios de cinéma, par leurs décors théâtraux ${ }^{27}$, Mayer voyait la possibilité de faire émerger un autre type de film, qui capterait le mouvement de la foule et de la circulation, les rythmes, les tempi et la musicalité de la vraie vie quotidienne dans la métropole. Partageant cette même passion pour une description improvisée et spontanée de l'existence quotidienne urbaine, Karl Freund, le collaborateur et caméraman de Mayer, commença à

24. - Pour plus d'approfondissements sur Ruttmann et ses films, voir Michael Cowan, Walter Ruttmann and the City of Multiplicity : Avant-Garde-AdvertisingModernity, Amsterdam, Amsterdam UP, 2014.

25. - Mark Shiel, Tony Fitzmaurice (éd.), Screening the city, London, Verso, 2003, p. 27.

26. - Siegfried Kracauer, De Caligari à Hitler. Une histoire psychologique du cinéma allemand, Paris, Flammarion, 1987, p. 202.

27. - Comme le montre bien l'exemple du film Le Cabinet du docteur Caligari. Cela est peut-être une raison pour laquelle Mayer souhaitait que son nom soit supprimé du générique du film de Wiene. 
tourner subrepticement des séquences dans les rues, en captant la ville pour ainsi dire à son insu, afin de dévoiler le vrai Berlin et les véritables Berlinois. Walter Ruttmann qui avait élaboré dans les années 1920 ses propres courts-métrages formalistes d'inspiration géométrique (Opus I et $I I)$ a été amené par Mayer et Freund à réaliser, composer et monter le matériau ainsi enregistré. Travaillant étroitement avec le compositeur autrichien Edmund Meisel, Ruttmann cherchait à représenter les multiples figures et rythmes de la ville moderne au moyen d'images en mouvement insérées dans un montage rapide et synchronisées avec la partition musicale. Le visuel et l'acoustique devaient fonctionner simultanément, dans un souci d'accentuation et parfois de contraste, en variant les humeurs et les moments, par exemple en utilisant les percussions et les instruments à vent pour intensifier les séquences en mettant en scène le fonctionnement dynamique des machines.

Dans un article pour la Berliner Zeitung am Mittag, Ruttmann affirme que son objectif « [...] est de construire à partir d'un matériau vivant, de créer une symphonie urbaine à partir des millions d'énergies présentes dans la grande ville en tant qu'organisme [...] $»^{28}$. Dans un article de 1927 paru dans le magazine Filmspiegel, il développe quatre principes de base relatifs à cette tentative : « [...] la réalisation cohérente de la rythmique musicale $»^{29}$ du montage (à noter, car c'est révélateur, non pas du matériau lui-même) ; la recherche d'immédiateté au moyen de la technique de la caméra cachée, faisant écho à la conception de Freund ; l'autosuffisance visuelle, c'est-à-dire que les intertitres ou les commentaires étaient superflus; l'accent était mis sur « l'abstrait, le géométrique », les formes et les schémas. En élargissant et en élaborant la réflexion sur les quatre préceptes de Ruttmann, je propose de mettre en avant ce qui est particulièrement révélateur pour ses films et de rappeler la définition des notions suivantes ${ }^{30}$ : La première notion est celle d'avant-gardisme, qui constitue une expérimentation consciente et innovante en termes de formes cinématographiques, techniques et technologies. Le film de Ruttmann joue avec les structures narratives conventionnelles, les angles de prise de vue et les pratiques de montage. La ville dépeinte est sujette à un processus de défamiliarisation de telle façon que le public voit renaître ses espaces, ses structures et ses habitants. La caméra révèle ce que les citadins ordinaires, en tant que passants occupés et distraits, ne parviennent pas à percevoir par manque

28. - M. Shiel, T. Fitzmaurice (éd.), Screening the city, op. cit., p. 43.

29. - Ibid.

30. - En effet, et dans le paradigme des multiples films de symphonies urbaines qui en résultaient comme São Paolo. A Metropolitan Symphony (Adalberto Kemeny/ Rudolf Rex Lustig, 1929, 90 min.) et Lisboa (José Leitão de Barros, 1930, 88 min.) 
de temps, d'attention ou d'intérêt ${ }^{31}$. D'une façon typique, le film est hautement autoréférentiel, c'est-à-dire que le travail sur la substance du film même, le dispositif de la caméra, de l'œil, de l'optique est intégré dans les séquences elles-mêmes, d'une façon fréquente et explicite. Ruttmann ne laisse passer aucune occasion de démontrer les affinités électives entre le paysage urbain moderne (des gens et des choses pris dans un mouvement constant) et le médium cinématographique (le film).

Une seconde notion est celle de la synchronisation acoustique et optique : l'impact du film comme un genre de Gesamtkunstwerk (œuvre d'art totale) résidait exclusivement dans la combinaison et dans l'interaction des images urbaines et d'une bande-son musicale extra-diégétique. La bande-son n'est pas un simple fond sonore mais sert à mettre en valeur ce qui est montré à l'écran, apportant un complément à son atmosphère, intensifiant les rythmes du mouvement, la vitesse et le dynamisme, fournissant du contraste et du contrepoint. Le film cherchait à créer une expérience esthétique d'un genre particulier, en accord avec cette vision énergique, vibrante de la ville moderne : choc, dissonance, désorientation, agression sensorielle, fragmentation. Le mot symphonie n'est peut-être pas toujours le terme approprié, on dirait plutôt diaphonie, cacophonie. Dans tous les cas, rien ne devait détourner de l'interaction entre partition et image. En conséquence les vrais sons de la ville (le bruit de la circulation et des machines, le tohu-bohu des conversations dans les bars et ainsi de suite), étaient exclus, et il n'y avait aucun rôle pour une quelconque voix-off de l'auteur donnant des explications et des commentaires d'ordre politique.

Une troisième notion est celle de dynamisme : le film de Ruttmann met l'accent sur l'environnement métropolitain comme étant caractérisé par le mouvement spatial et le changement temporel, et, par-dessus tout, par le rythme. Les images représentent de façon ininterrompue le mouvement et la vitesse des machines et de la circulation, le rythme rapide des pas des piétons et les bousculades des voyageurs. Partout dans la ville, il y a des constructions, des démolitions, des réaménagements et des régénérations comme si le tissu urbain lui-même était constamment sujet à un renouveau. L'interaction des humains avec la première et seconde nature est toujours énergique, répétitive et cinétique. Peu de scènes représentent des moments de calme et de repos, mais celles-ci fonctionnent principalement comme de brefs interludes ou comme un contrepoint au tempo accéléré des séquences de clôture. La ville vit en accéléré presque toujours et partout, allegro. En effet, Berlin n'est jamais seulement une ville, elle est en devenir perpétuel, se réinventant et se recomposant tous les jours et à tous les moments. La seule constante

31. - En documentant ce qui nous a rendu indifférents et aveugles, le film de Ruttman présente à son auditoire (du moins dans une certaine mesure) ce que Walter Benjamin (1936) appelait l'inconscient optique du quotidien. 
est la pulsation de la ville, son rythme, qui accélère ou ralentit, mais ne s'arrête jamais, pas même pour reprendre son souffle.

La dernière notion, celle d'impersonnalité : la ville elle-même étant au centre du film de Ruttmann, les personnages humains et les histoires personnelles sont peu de choses, servant parfois seulement à ouvrir sur des aspects de l'habitat urbain qui autrement passeraient inaperçus. Au lieu de cela, les humains apparaissent sous deux aspects principaux : en tant que «types », se positionnant comme figures représentatives de classes sociales, de groupes religieux particuliers, de groupes ethniques, ou en tant que collectivités : consommateurs faisant du lèche-vitrine, clientèle de café, public des théâtres et des cinémas, clients dans les grands magasins, passants dans les rues, sur les places et les marchés, passagers dans le système des transports en commun. L'arc narratif principal est alors fourni non pas tant par une histoire singulière, particulière et individuelle que par le déploiement partagé d'un jour ordinaire dans la vie de la ville et des citadins. La symphonie du film de Ruttmann ne met pas en avant une personne en particulier. La vie métropolitaine est imaginée comme un phénomène de masse.

Kracauer lui-même fit partie des détracteurs le plus déterminés, et de ceux qui critiquèrent de façon pénétrante le film Berlin au moment de sa sortie. Cela est quelque peu surprenant : on s'attendait à ce qu'il manifeste un peu plus d'enthousiasme pour le film de Ruttmann ${ }^{32}$. Il est vrai que Kracauer avoue rétrospectivement que Berlin est « un film extrêmement important $»^{33}$. Mais c'est seulement à cause de son statut d'exemple-type de l'« expression superficielle », c'est-à-dire en tant que symptôme de la neutralité apolitique du cinéma allemand sous l'influence de la Nouvelle Objectivité à la fin des années 1920. Pour Kracauer, le film était une combinaison parfaite entre prétention et superficialité. Que Carl Mayer lui-même se soit retiré du projet de la symphonie de la ville au vu de la forme qu'il prenait avec Ruttmann est parlant pour Kracauer. Mayer regrettait qu'au lieu de pénétrer dans le « vrai », le travail minutieux de montage et l'insistance sur la primauté du rythme des images se faisaient au détriment du matériau lui-même, réduisant le film à un simple inventaire superficiel. En privilégiant et en donnant la priorité à un travail artistique sur les angles de prise de vue, le montage rythmé, Ruttmann était davantage intéressé par les techniques de composition et d'esthétique formaliste, c'est-à-dire les schémas géométriques, les objets

32. - Après tout Kracauer lui-même était fasciné par les affinités électives entre le film et le tourbillon d'activité de la ville contemporaine. La redécouverte de cet environnement, des qualités du réel, en surmontant le regard fatigué, indifférent, habitué du citadin moderne, allait ainsi être au centre de ce qui est pour le Kracauer de la Théorie du film la tâche du cinéma, c'est-à-dire la rédemption de la réalité physique comme il l'indique dans le sous-titre incompris de Theory of film de 1960.

33. - S. Kracauer, De Caligari à Hitler, op. cit., p. 202. 
et les structures, les gradations d'ombres et de lumière, la synchronisation entre le son et l'image, que par la révélation authentique du désordre et des significations de l'existence urbaine quotidienne elle-même ${ }^{34}$.

Kracauer observe à titre d'exemple que les machines tournent, mais que rien n'est dit du sens de leur fonction ou de leur relation à l'industrie ou la production. Faut-il y voir un panégyrique de l'inventivité du capitalisme technologique ou un témoignage de la réification et l'aliénation des travailleurs ? Vitesse, tempo, accélération, ces abstractions sont présentées et célébrées pour elles-mêmes. Ansi, malgré les ambitions affichées par Ruttmann en matière de montage rythmique, les diverses images et séquences demeurent détachées et disparates, elles échouent précisément à créer ces liens essentiels entre différents phénomènes qui pourraient leur donner une véritable signification et une raison d'être. Simplement juxtaposés (nebeneinander), et démunis de commentaires et convictions, ces éléments de la vie quotidienne sont contigus, mais inertes. Ils ne nous disent rien, ou plutôt rien de particulier ${ }^{35}$. La symphonie urbaine n'éclaire pas le réel mais plutôt sa vision artificiellement embellie et sa mise en valeur poétique. Cette ville n'est pas celle qui est expérimentée de façon courante et quotidienne, elle se présente plutôt comme un ornement parfaitement lisse. Le film de Ruttmann est un triomphe de l'abstraction (Abstraktheit). Pour quelles raisons ce film est-il un tel échec ? Kracauer ne mâche pas ses mots :

Parce que ces messieurs n'avaient aucunement l'ambition de montrer une grande ville telle qu'elle est en réalité, mais ils avaient plutôt

34. - « [...] images en partie excellentes » affirme Kracauer dans sa critique du film Berlin en 1927, avec des « superpositions habiles », un film « techniquement irréprochable » et « qui n'est pas dépourvu d'imagination visuelle», «[...] mais s'agitil de Berlin »? Il fournit lui-même la réponse : "Berlin ne se donne aucunement à voir ». Siegfried Kracauer, "Wir schaffens », in : Id., Schriften, Bd. 6.1, Frankfurt a.M., Suhrkamp Verlag, 2004, p. 411-413, ici p. 411. Au regard de ce que Kracauer a développé plus tard dans sa Théorie du film (1960), on pourrait dire que la question de la «tendance formative » du film (les idées préconçues et les interventions créatives des réalisateurs eux-mêmes) était devenue une priorité et avait pris le dessus sur le matériau concret sur lequel Mayer et Freund avaient mis l'accent : les choses de la vie ordinaire, l'accent sur la tendance réaliste dans le film.

35. - Dans De Caligari à Hitler, Kracauer prend la liberté de citer son propre verdict sur le film à partir d'un essai publié dans le Frankfurter Zeitung : « Ruttmann, au lieu de pénétrer dans son immense sujet avec une véritable compréhension de ses structures sociale, économique et politique [...], Ruttmann enregistre des milliers de détails sans les relier entre eux, ou au mieux les relie par des transitions fictives vides de tout contenu. Son film est peut-être fondé sur l'idée de Berlin en tant que ville de rythme et de travail ; mais il s'agit là d'une idée formelle qui n'implique pas d'autre contenu et peut-être pour cette raison, enivre de vie réelle et de littérature la mesquine bourgeoisie allemande. Cette symphonie échoue à montrer quelque chose, parce qu'elle ne recouvre aucun contexte significatif », S. Kracauer, De Caligari à Hitler, op. cit., p. 209. 
l'ambition démesurée de composer d'emblée une grande symphonie urbaine. C'étaient de mauvais compositeurs ${ }^{36}$.

La superficialité et l'abstraction (Abstraktheit) ont de l'importance pour Kracauer parce qu'en dépit des intentions déclarées des réalisateurs de $n e$ pas être non-critique, c'est précisément cela que devient Berlin : politiquement quiétiste. Kracauer était exaspéré par la naïveté politique du film ${ }^{37}$, par sa « neutralité ambiguë» et « atone » ${ }^{38}$ : les images mettant en scène les riches, des « vainqueurs » dînant dans les restaurants luxueux, s'entrecoupent avec celles des pauvres, affamés, et mangeant de maigres repas, mais il n'y pas de questionnement sur le pourquoi, pas de doigt accusateur désignant les responsables d'une telle pénurie au milieu de l'abondance. Qui est à blâmer ? Que faut-il faire ? Kracauer suggère que cette abdication politique fondamentale de la part de Ruttmann est la raison pour laquelle Mayer prit la décision de quitter le film ${ }^{39}$. C'est aussi ce qui rend Berlin si important et si représentatif de son temps.

\section{L'orchestration de la masse}

Si Berlin, symphonie d'une grande ville se trouve à l'intersection de L'Ornement de la masse et des Employés, il a également un lien direct avec Totalitäre Propaganda. Tout d'abord, le film peut être vu comme une version cinématographique des Employés. En se préoccupant de la vie quotidienne des citadins, le film porte inévitablement son attention sur les classes représentatives de la ville : pour l'essentiel le monde des cols blancs, avec ses habitudes, ses pratiques, ses modes, ses lieux, ses usages. Ils sont présentés comme des individus consommant dans les cafés, passant devant les marchandises et les magasins, allant au cinéma et assistant aux pièces de théâtre. Le film de Ruttmann enregistre de façon implicite la vie quotidienne de cette classe spécifiquement urbaine. En effet, il n'est pas une mise en images par anticipation du livre, mais c'est le livre de Kracauer qui constitue une réponse à toutes les insuffisances du film, liées à l'absence de toute critique, à l'incapacité navrante du réalisateur de mettre au jour un contexte significatif. Tout se passe comme si l'étude de Kracauer était un rectificatif critique du film de Ruttmann. Les Employés est dans le domaine de la littérature ce que la symphonie urbaine aurait pu être dans le domaine cinématographique.

Le lien du film avec « L'ornement de la masse » est à la fois explicite et parlant. Dans l'acte V, les Tiller Girls font leur apparition dans la symphonie urbaine de Ruttmann. Strathausen avance que Berlin est une

36. - S. Kracauer, « Wir schaffens », op. cit., p. 412.

37. - S. Kracauer, De Caligari à Hitler, op. cit., p. 208.

38. - Ibid.

39. - Ibid 
célébration de l'ornement de masse ${ }^{40}$. Je voudrais aller plus loin sur ce point : le film Berlin, symphonie d'une grande ville constitue lui-même un ornement de la masse. Il exprime les mêmes tendances que les Tiller Girls, la déshumanisation, la fragmentation, la rationalisation et la mécanisation. Le formalisme du film correspond aux corps disloqués et aux membres synchronisés de la troupe de danse souvent en musique, selon des lignes géométriques et des schémas abstraits. Le rythme rapide des images que Ruttmann considérait comme décisif pour la symphonie de la grande ville n'est rien d'autre que le rythme même de la Ratio, auquel obéissent les mouvements des jambes et des pieds des Tiller Girls.

L'architecture éphémère des foules, dans laquelle les membres qui la constituent sont assujettis aux multiples recompositions, et les configurations éphémères qu'ils forment sont soumises à des rythmes et des mouvements réguliers et entraînants : tout cela est fondamental pour Ruttmann. La chorégraphie corporelle de l'ornement de la masse trouve finalement son corollaire dans son orchestration urbaine (via le latin à partir du grec : orkhèstra, de orkheisthai : danser). L'impact visuel et corporel de tels assemblages et images n'a pas échappé à Ruttmann. Sept ans après seulement le film Berlin..., il allait travailler côte à côte avec Leni Riefenstahl sur Triomphe de la volonté (1935), en mettant les dernières techniques et technologies cinématographiques au service de la propagande national-socialiste.

La mise en scène des défilés et de l'apparat - véritable montage d'ornements de la masse en marche - mobilisera pleinement ses talents de précision et de synchronisation. Il allait travailler avec Riefenstahl une nouvelle fois sur Olympia (1938), un autre éloge des masses et des corps en mouvement soigneusement choisis. À ce moment-là, il avait déjà été scénographe pour le film de 1933 : Sang et sol, fondement du nouveau Reich (Blut und Boden - Grundlagen zum neuen Reich). Il allait enfin mourir de ses blessures sur le tournage de films de propagande de guerre sur le front, en juillet 1941. Berlin, symphonie d'une grande ville était en partie un apprentissage de l'esthétique de la propagande totalitaire, un apprentissage de « l'esthétisation de la politique».

Kracauer est sûrement parmi les premiers à s'appuyer sur l'« Euvre d'art à l'époque de sa reproductibilité technique » (1936) de Walter Benjamin ${ }^{41}$. Publié d'abord en français dans Zeitschrift für Sozialforschung, journal dans lequel Totalitäre Propaganda devait paraitre mais ne fut jamais publié. Nous pouvons considérer l'étude de Kracauer comme une tentative d'élaborer la conclusion énigmatique de Benjamin, dans laquelle il critique « l'esthétisation de la politique opérée par les doctrines totalitaires » et insiste sur le fait que « les forces

40. - M. Shiel, T. Fitzmaurice (éd.), Screening the city, op. cit., p. 32.

41. - S. Kracauer, Totalitäre Propaganda, op. cit., p. 80. 
constructives de l'humanité y répondent par la politisation de l'art $»^{42}$. Si cela est le cas, alors la compréhension que Kracauer a des masses et de l'ornement de la masse qui a été évoqué plus haut nous livre un rectificatif subtil mais non moins éloquent en nous orientant vers les notions de corporelles et de rythmiques. De façon ironique, il nous alerte sur le caractère non spectaculaire du spectacle. La propagande totalitaire n'était pas seulement vue sur les écrans ou entendue à la radio, elle était ressentie. C'est cette réalité physique qui exige un petit ajustement de la fameuse formule de Benjamin : le fascisme implique la synesthétisation de la politique, (je suis tenté de dire anesthétisation de la politique) auquel le communisme répond avec la politisation de la synesthésie.

Traduit de l'anglais par Antonella Di Trani et Olivier Agard

42. - Walter Benjamin, «L'œuvre d'art à l'époque de sa reproductibilité technique », in : Zeitschrift für Sozialforschung, 5, 1936, p. 40-68, ici p. 66. 